\title{
DIAGNÓSTICO SOBRE EL DECOMISO DE FAUNA SILVESTRE EN EL DEPARTAMENTO DEL CHOCÓ (PACÍFICO NORTE COLOMBIANO)
}

\section{DIAGNOSIS ON ILLEGAL SEIZURE OF WILDLIFE IN THE DEPARTMENT OF CHOCÓ (COLOMBIAN NORTHERN PACIFIC)}

\begin{abstract}
Jeferson Asprilla-Perea ${ }^{1}$, Jorge Eliecer Serna-Agudelo ${ }^{2}$, Yacira Palacios-Asprilla ${ }^{3}$
${ }^{1}$ Magister en Manejo y Conservación de Recursos Naturales, profesor Facultad de Ciencias Básicas, Universidad Tecnológica del Chocó "Diego Luís Córdoba”. Dirección: Cr. 13a N. 18-150 Quibdó, Chocó, Colombia. E-mail: jasprilla@gmail.com; ${ }^{2} E s-$ tudiante Facultad de Ciencias Básicas, Universidad Tecnológica del Chocó "Diego Luís Córdoba”. E-mail: jorgeserna123@ gmail.com; ${ }^{3}$ Estudiante Facultad de Ciencias Básicas, Universidad Tecnológica del Chocó "Diego Luís Córdoba". E-mail: jharykyiseth@yahoo.es
\end{abstract}

Rev. U.D.C.A Act. \& Div. Cient. 16(1): 175 - 184, 2013

\section{RESUMEN}

El departamento del Chocó (Pacífico norte colombiano) figura dentro de los ocho lugares registrados como los principales sitios de origen de la fauna silvestre decomisada en Colombia; sin embargo, para la región no se cuenta con estadísticas claras sobre las especies con mayor frecuencia de decomiso, ni con evaluaciones sobre el proceso y la disposición de los ejemplares posterior al mismo, elementos que podrían ser fundamentales para el diseño de estrategias de conservación del recurso. Por lo anterior, en el presente estudio, se identificó la fauna silvestre decomisada en el departamento del Chocó, durante 2005 a 2010, para lo cual, se revisaron las bases de datos oficiales de autoridades ambientales y se entrevistaron a funcionarios de las mismas. A nivel de resultados, se obtuvo que las aves y los mamíferos son los grupos taxonómicos más decomisados, ya que cerca del $80 \%$ de individuos aprehendidos pertenecen a estos taxones. La especie más incautada fue Trachemys callirostris callirostris (tortuga Icotea), con el 22,2\% (161 individuos), del total de individuos. El municipio de Quibdó es la zona geográfica del departamento donde se registró el mayor número de individuos y de especies decomisadas. No se identificaron rutas establecidas para el tráfico de fauna silvestre. En cuanto a conservación, se estableció que la mayor parte de las especies decomisadas (80,6\%), se clasifican en alguna categoría de amenaza, según los criterios establecidos por la Unión Internacional para la Conservación de la Naturaleza
(UICN), siendo los peces, las aves y los mamíferos los vertebrados más críticos en esta materia.

Palabras clave: Especies faunísticas decomisadas, autoridades ambientales, conservación.

\section{SUMMARY}

Chocó department belongs to the eight places with the major illegal wildlife commercial trade and wildlife confiscation registered in Colombia. Nevertheless, there is no clear statistic about species frequently involved; a serious assessment about wildlife decommissions (processes and management) have not been carried out. This Information could be used as tool to support implementation of resource management or as conservation strategy. This study describes seized wildlife in the department of Chocó during six years (2005-2010), based on an intensive revision from official databases of environmental authorities and interviews with officials. The results showed that $80 \%$ of the seized wildlife is composed of birds and mammals. Trachemys callirostris callirostris (turtle Icotea) was the most seized species (161 individuals corresponding to $22.2 \%$ of illegal wildlife seized). The municipality of Quibdó is the geographical area of Chocó department where the highest number of individuals and species were confiscated. No established routes for illegal wildlife trafficking were found. In terms of conservation the majority of confiscated species $(80.6 \%)$ are in a category of threat according to crite- 
ria established by the International Union for Conservation of Nature (IUCN) Red List of threatened species. Fish, bird and mammal species seized in Chocó department are under the highest threatened level by the UICN criteria.

Key words: Illegal wildlife seizure, environmental authorities, conservation.

\section{INTRODUCCIÓN}

El territorio de Colombia contiene más del 10\% de la biodiversidad mundial faunística (Mayr Maldonado, 1999). Se estima que en el país existen aproximadamente 45.000 especies de plantas vasculares, 1.875 de aves, 733 de anfibios, 520 de reptiles y 447 de mamíferos (Rodríguez-Mahecha et al. 2006). Entre el patrimonio natural de Colombia, la diversidad de fauna silvestre constituye uno de los componentes de mayor significado. A nivel global, esta riqueza sitúa a Colombia, en el primer lugar, en número de especies de aves, en el segundo, respecto a anfibios y, en el tercero, con primates, reptiles y mariposas (Rangel, 2004).

Dentro del territorio nacional, la fauna de vertebrados del Chocó Biogeográfico se encuentra representada por 132 especies de mamíferos, 578 de aves, 139 de reptiles, 92 de anfibios (Rangel, 2004).

Esta enorme riqueza de fauna silvestre constituye un recurso determinante en la dinámica natural de los ecosistemas, al igual que para la subsistencia de las comunidades humanas de las áreas rurales del neotrópico; sin embargo, es importante señalar que las actividades extractivas no planificadas de un elevado número de animales y de manera regular constituyen un gran riesgo para la conservación de las especies (Baptiste et al. 2002; Bennett \& Robinson, 2000; Redford, 1992; Robinson \& Redford, 1991).

Por lo anterior y debido a que la protección de dicha biodiversidad está a cargo del Estado, el cual, por mandato constitucional tiene el deber de garantizar la sostenibilidad de la diversidad biológica del país, ha establecido diferentes acciones educativas, normativas y de planificación en pro de la conservación del patrimonio diverso colombiano.

A nivel normativo, se han diseñado medidas preventivas y sancionatorias, entre las que se encuentra el decomiso, concebido como una estrategia importante para contribuir a la conservación de las especies removidas de sus hábitats naturales, reduciendo el tráfico ilegal (Ley 99 de 1993, Ley 1333 de 2009, Resolución 2064 de 2010 del Ministerio de Ambiente, Vivienda y Desarrollo Territorial); no obstante, y pese a que el departamento del Chocó figura entre los ocho lugares registrados como el principal sitio de origen de la fauna silvestre decomisada en Colombia (Contraloría Gene- ral de la República, 2005), aun no se cuenta con estadísticas claras para la región sobre las especies con mayor frecuencia de decomiso, ni con evaluaciones sobre el proceso de tráfico y disposición de los ejemplares posterior al mismo, elementos que son fundamentales para el diseño de estrategias de conservación del recurso faunístico y para el fortalecimiento de las acciones adelantadas por las autoridades ambientales, en el cumplimiento de sus funciones de vigilancia y de control, ya que según Baptiste et al. (2002), el total de los decomisos puede estar entre el 1 y el $10 \%$ de la fauna silvestre comercializada.

En el presente estudio evaluamos diferentes aspectos relacionados con la fauna silvestre decomisada en el departamento del Chocó, como un aporte de conocimientos para la identificación de posibles especies en riesgo por su tenencia ilegal, con la finalidad de proveer elementos que ayuden a las autoridades ambientales en el cumplimiento de sus actividades de vigilancia y control sobre este recurso.

\section{MATERIALES Y MÉTODOS}

Área comprendida en el análisis. El área de influencia del proyecto fue el departamento del Chocó (Colombia), para lo cual, se revisaron las bases de datos de decomiso de fauna silvestre de la Corporación Autónoma Regional para el Desarrollo Sostenible del Chocó "CODECHOCO" y del Instituto Colombiano de Desarrollo Rural "INCODER". Debido a la disponibilidad de datos por parte de las autoridades ambientales, se estableció un rango temporal de seis años de evaluación para el análisis (2005 - 2010).

Con los datos obtenidos, se conformó una lista sobre la composición taxonómica de especies de fauna silvestre decomisada; igualmente, se analizan el número de especies e individuos decomisados por año, datos que son presentados gráficamente y en tablas, para facilitar su comprensión.

Diferencias estadísticamente significativas entre el número de individuos registrados por año para las diez especies principales de fauna silvestre decomisada en el Chocó fueron evaluadas, a través de un Análisis de Varianza de una vía. Este mismo procedimiento, se realizó para detectar diferencias estadísticamente significativas entre el número de individuos decomisados por año en cada uno de los ocho municipios del departamento del Chocó, donde se documentó la mayor cantidad de incautaciones. Los datos fueron analizados usando el paquete estadístico STATGRAPHICS Centurion XV.

Debido a que los datos de decomisos reportados para el bocachico, Prochilodus magdalenae corresponden a una temporalidad diferente a los obtenidos para las otras especies de vertebrados analizadas y a que es un taxón cuyo aprovechamiento extractivo, con fines comerciales, es aceptado por 
las autoridades ambientales, el análisis de sus decomisos se llevó a cabo de manera independiente.

Evaluación del estado de conservación. Una vez determinada la composición taxonómica de las principales especies de fauna silvestre decomisada por parte de autoridades ambientales en el departamento del Chocó, se efectuó una revisión bibliográfica para la determinación del estado de amenaza para las poblaciones colombianas, de cada una de las especies identificadas. Este proceso, se realizó de acuerdo a las categorías establecidas por la Unión Internacional para la Conservación de la Naturaleza "UICN" (Castaño-Mora, 2002; Mojica et al. 2002; Resolución 0572 de 2005 del Ministerio de Ambiente, Vivienda y Desarrollo Territorial).

\section{RESULTADOS Y DISCUSIÓN}

Composición taxonómica de especies decomisadas (2005 2010). Se registró un total de 724 individuos de fauna silvestre decomisados durante los seis años de evaluación. Dichos individuos pertenecen a cuatro clases (peces, reptiles, aves y mamíferos), 25 órdenes, 51 familias, 69 géneros y 72 especies (Tabla 1).

La clase aves presenta el mayor número de órdenes, familias, géneros y especies decomisadas (48\%, 39,2\%, 43,5\% y $45,8 \%$, respectivamente), mientras que la clase peces fue la menos representada (Tabla 1). Estos resultados son concordantes con lo reportado por Baptiste et al. (2002), en sus investigaciones sobre el tráfico de fauna silvestre en Colombia, quienes mencionan que las exportaciones ilegales de animales silvestres en el país se concentran en las aves, en 79,83\%.

Pese a lo anterior, los resultados del presente estudio difieren con los obtenidos por Cruz-Antía et al. (2010), para el municipio de Puerto Carreño, en el departamento del Vichada, donde se reporta que durante el 2008 y el 2009, los mamíferos fueron el grupo taxonómico con mayor porcentaje de especies decomisadas, con el 58\% del total registrado (11 especies), mientras que las aves estuvieron representadas solamente en un $26 \%$ ( 5 especies) y los reptiles, con el 16\% (3 especies).

Comportamiento del decomiso de P. magdalenae (bocachico) entre los años 2004 - 2007. Los registros de decomiso del bocachico, durante los años de evaluación, muestran un total de 88 toneladas incautadas de este pez, lo cual, corresponde a 338.461 individuos de la especie, que equivalen a 6.769 arrobas (entendiéndose en el concepto tradicional de la región, una arroba como 50 individuos, aproximadamente).

La dinámica de decomisos muestra que para el 2004 y 2005, se registraron los mayores valores en términos de toneladas de bocachico incautado, ya que del $74 \%$ (65 toneladas), del total aprehendido, un 26\%, se decomisó entre el 2005 y el 2007.

Es preciso señalar que pese a la importancia socio-económica del bocachico para el Chocó, la información suministrada por el Instituto Colombiano para el Desarrollo Rural (INCODER, 2010) no permitió identificar las causas asociadas a esta considerable disminución en decomisos de esta especie, entre el 2006 y el 2007.

Principales especies de fauna silvestre decomisada. La especie que presentó el mayor porcentaje de decomiso durante el 2005 y 2010 fue Trachemys callirostris callirostris (tortuga Icotea), con el 22,2\% (equivalentes a 161 individuos), del total registrado (724), seguido de Serinus canaria (canario) y Melopsittacus undulatus (perico australiano), con 6,5\% y $5,7 \%$, respectivamente (Tabla 2 ).

Es pertinente anotar que las especies $S$. canaria y M. undulatus son exóticas en el departamento del Chocó, por tanto, su presencia, en la mayoría de los casos, obedece al tráfico ilegal. Este hecho, se puede verificar al revisar en las actas

Tabla 1. Número de individuos de fauna silvestre (por grupos taxonómicos) decomisada en el departamento del Chocó (2005 - 2010).

\begin{tabular}{|c|c|c|c|c|c|c|c|c|}
\hline Clase & Orden & $\mathbf{\%}$ & Familia & $\mathbf{\%}$ & Género & \% & Especie & $\begin{array}{c}\text { Porcentaje } \\
\text { (\%) }\end{array}$ \\
\hline Peces & 2 & 8,0 & 2 & 3,9 & 2 & 2,9 & 2 & 2,8 \\
\hline Reptiles & 3 & 12,0 & 11 & 21,6 & 13 & 18,8 & 13 & 18,1 \\
\hline Aves & 12 & 48,0 & 20 & 39,2 & 30 & 43,5 & 33 & 45,8 \\
\hline Mamíferos & 8 & 32,0 & 18 & 35,3 & 24 & 34,8 & 24 & 33,3 \\
\hline Total & 25 & 100 & 51 & 100 & 69 & 100 & 72 & 100 \\
\hline
\end{tabular}

Fuente: Elaboración propia con datos suministrados por CODECHOCO, 2010. 
Tabla 2. Porcentaje de especies de fauna silvestre decomisada en el departamento del Chocó (2005 - 2010) y su categoría de amenaza, según la Unión Internacional para la Conservación de la Naturaleza (UICN).

\begin{tabular}{|c|c|c|c|c|}
\hline $\begin{array}{l}\text { NOMBRE } \\
\text { CIENTÍFICO }\end{array}$ & $\begin{array}{l}\text { NOMBRE } \\
\text { COMÚN }\end{array}$ & $\begin{array}{l}\text { NÚMERO TOTAL DE } \\
\text { INDIVIDUOS (2005 - 2010) }\end{array}$ & PORCENTAJE & $\begin{array}{l}\text { AMENAZA } \\
\text { (UICN) }\end{array}$ \\
\hline $\begin{array}{l}\text { Trachemys callirostris calliro- } \\
\text { stris }\end{array}$ & Tortuga icotea & 161 & 22,2 & $\mathrm{VU}$ \\
\hline Serinus canaria & Canario & 47 & 6,5 & LC \\
\hline Melopsittacus undulatus & Perico australiano & 41 & 5,7 & LC \\
\hline Amazona ochrocephala & Loro frentiamarillo & 31 & 4,3 & LC \\
\hline Bradypus variegatus & Perezoso & 31 & 4,3 & LC \\
\hline Kinosternon dunni & Tortuga tapa culo & 30 & 4,1 & $\mathrm{VU}$ \\
\hline Potos flavus & Cuzumbo & 28 & 3,9 & LC \\
\hline Amazona farinosa & Loro real & 25 & 3,5 & LC \\
\hline Boa constrictor & Boa & 23 & 3,2 & \\
\hline Cuniculus paca & Guagua & 23 & 3,2 & LC \\
\hline Brotogeris jugularis & Perico real & 20 & 2,8 & LC \\
\hline Saguinus leucopus & Mico titi & 20 & 2,8 & EN \\
\hline Bubo virginianus & Buho real & 16 & 2,2 & LC \\
\hline Ateles geoffroyi rufiventris & Mico marimonda & 14 & 1,9 & EN \\
\hline Cacatua sp & Cacatua & 14 & 1,9 & \\
\hline Amazona autumnalis & Loro frentirojo & 13 & 1,8 & LC \\
\hline Choloepus hoffmanni & $\begin{array}{l}\text { Perezoso de dos } \\
\text { uñas }\end{array}$ & 13 & 1,8 & LC \\
\hline Iguana iguana & Iguana & 12 & 1,7 & \\
\hline Pionus menstruus & Loro cabeciazul & 12 & 1,7 & LC \\
\hline Cebus capucinus & Mono cariblanco & 9 & 1,2 & LC \\
\hline Ara ararauna & Guacamaya & 8 & 1,1 & LC \\
\hline Dasyprocta punctata & Guatin & 8 & 1,1 & LC \\
\hline Pseudoscops clamator & Buho & 8 & 1,1 & LC \\
\hline Columba sp & Palomas & 7 & 1,0 & \\
\hline Penelope ortoni & Pava de monte & 6 & 0,8 & EN \\
\hline Porphyrio martinica & Chilaco & 6 & 0,8 & LC \\
\hline Sciurus granatensis & Ardilla coliroja & 6 & 0,8 & LC \\
\hline Didelphis marsupialis & Chucha & 5 & 0,7 & LC \\
\hline Chelonoidis carbonaria & Tortuga morro & 5 & 0,7 & \\
\hline Mimus gilvus & Sinsonte & 5 & 0,7 & LC \\
\hline Tamandua tetradactyla & Oso hormiguero & 5 & 0,7 & LC \\
\hline Chironius carinatus & Culebra cazadora & 4 & 0,6 & \\
\hline Crax rubra & Paujil & 4 & 0,6 & $\mathrm{VU}$ \\
\hline Dasypus novemcinctus & Armadillo & 4 & 0,6 & LC \\
\hline Eremophila alpestris peregrina & Alondra & 4 & 0,6 & LC \\
\hline Megascops choliba & Currucutú & 4 & 0,6 & LC \\
\hline Ramphastos swainsonii & Tucan & 4 & 0,6 & LC \\
\hline Caiman crocodilus fuscus & Babilla & 3 & 0,4 & \\
\hline
\end{tabular}


Continuación tabla 2

\begin{tabular}{|c|c|c|c|c|}
\hline $\begin{array}{l}\text { NOMBRE } \\
\text { CIENTÍFICO }\end{array}$ & $\begin{array}{l}\text { NOMBRE } \\
\text { COMÚN }\end{array}$ & $\begin{array}{c}\text { NÚMERO TOTAL DE } \\
\text { INDIVIDUOS (2005 - 2010) }\end{array}$ & PORCENTAJE & $\begin{array}{l}\text { AMENAZA } \\
\text { (UICN) }\end{array}$ \\
\hline Leopardus pardalis & Tigrillo & 3 & 0,4 & LC \\
\hline Psarocolius cassini & Mochileros & 3 & 0,4 & EN \\
\hline Aotus lemurinus & Marteja & 2 & 0,3 & $\mathrm{VU}$ \\
\hline Ara severa & Cotorra & 2 & 0,3 & \\
\hline Coendou bicolor & Eriso ó poerco & 2 & 0,3 & LC \\
\hline Galictis vittata & Grison & 2 & 0,3 & LC \\
\hline Gallinula chloropus & Polla de agua & 2 & 0,3 & LC \\
\hline Podocnemys sp & Tortuga charra & 2 & 0,3 & EN \\
\hline Proechimys semispinosus & Ratón espina & 2 & 0,3 & LC \\
\hline Pseudemys sp & Tortuga & 2 & 0,3 & \\
\hline Aburria aburri & Pava negra & 1 & 0,1 & NT \\
\hline Alouatta seniculus & Mono aullador & 1 & 0,1 & LC \\
\hline Anthus sp & Ave chilin & 1 & 0,1 & \\
\hline Arremon aurantiirostris & Pajaro pinzón & 1 & 0,1 & LC \\
\hline Buteo magnirostris magnirostris & Gavilán & 1 & 0,1 & LC \\
\hline Chelydra serpentina & Tortuga bache & 1 & 0,1 & LC \\
\hline Chironectes minimus & Chucha de agua & 1 & 0,1 & LC \\
\hline Chloroceryle sp & Martin pescador & 1 & 0,1 & LC \\
\hline Chordeiles acutipennis & Chota cabras & 1 & 0,1 & LC \\
\hline Epicrates cenchria & Boa arcoíris & 1 & 0,1 & \\
\hline Fregata magnificens & Tijereta & 1 & 0,1 & LC \\
\hline Icterus gálbula & Turpial & 1 & 0,1 & LC \\
\hline Leptophis ahaetulla chocoensis & Culebra & 1 & 0,1 & \\
\hline Micrurus sp & Serpiente coral & 1 & 0,1 & \\
\hline Myrmecophaga tridactyla & Oso hormiguero & 1 & 0,1 & $\mathrm{VU}$ \\
\hline Panthera onca & Tigre mariposa & 1 & 0,1 & NT \\
\hline Pecari tajacu & Zaino & 1 & 0,1 & LC \\
\hline Potamotrygon magdalenae & Raya & 1 & 0,1 & NT \\
\hline Sylvilagus brasiliensis & Conejo de monte & 1 & 0,1 & LC \\
\hline Tayassu pecari & Tatabro & 1 & 0,1 & NT \\
\hline Thraupis episcopus & Azulejo & 1 & 0,1 & LC \\
\hline Tigrisoma mexicanum & Garza manteca & 1 & 0,1 & LC \\
\hline Tyto alba & Lechuza & 1 & 0,1 & LC \\
\hline \multicolumn{2}{|c|}{ Total fauna silvestre (no bocachico) } & 724 & 100 & \\
\hline NOMBRE CIENTÍFICO & $\begin{array}{l}\text { NOMBRE } \\
\text { COMÚN }\end{array}$ & $\begin{array}{l}\text { NÚMERO TOTAL DE } \\
\text { INDIVIDUOS (2004 - 2007) }\end{array}$ & PORCENTAJE & $\begin{array}{l}\text { AMENAZA } \\
\text { (UICN) }\end{array}$ \\
\hline Prochilodus magdalenae & Bocachico & 338.461 & 100 & \\
\hline
\end{tabular}

de decomiso los datos de procedencia de los individuos, donde se valida que, generalmente, estas dos especies son procedentes de las ciudades de Cali y de Medellín. Dicha procedencia no hace referencia a la distribución geográfica de la especie, sino a la ciudad desde donde se trasladaron los ejemplares hacia el departamento del Chocó. 
Otras especies nativas con porcentaje de decomiso importante fueron: Bradypus variegatus (oso perezoso), Amazonas ochrocephala (loro frentiamarillo), Kinosternon dunni (tortuga tapaculo), Potos flavus (cusumbo, perrito de monte) y Amazonas farinosa (Loro real), con 4,3\%, 4,3\%, 4,1\%, $3,9 \%$ y $3,5 \%$, respectivamente (Tabla 2 ).

Se estableció que no existen diferencias estadísticamente significativas entre el número de individuos registrados por año para las diez principales especies decomisadas $(\mathrm{F}=$ 1,$89 ; \mathrm{P}=0,07 ; \alpha=0,05$ ), incluyendo $T$. c. callirostris (tortuga icotea), la cual, presentó el número de decomisos más elevado.

Los altos valores de decomiso reportados para la tortuga icotea en el periodo evaluado, es relacionado en este trabajo, con el planteamiento de Thorbjarnarson et al. (2000), quienes afirman que las tortugas, por ser una fuente de proteínas de fácil captura y de almacenamiento, han sido un importante elemento en la alimentación del hombre por milenios y los habitantes de zonas rurales, en diferentes partes del mundo, dependen en diferente grado de ellas y de sus productos, para cubrir sus necesidades básicas.

Número de individuos y especies de fauna silvestre decomisada por año. Se identificó que el 2009 presentó el mayor valor, con 216 individuos, los cuales, equivalen al $29,8 \%$ de la totalidad de ejemplares decomisados, durante los seis años de evaluación. De la misma manera, se observó que para el 2005, el número de decomisos fue solamente de 17 (el 2,3\% del total decomisado) (Figura 1).

En cuanto a número de especies decomisadas por año, el 2009 presentó el mayor valor, con el $69,4 \%$ del total registrado (50 especies), mientras que el 2005 fue el menor, representado con el 11,1\% (Figura 1).

Debido a que no se dispuso de datos sobre el número de operativos adelantados por las autoridades competentes por año en cada municipio, no podemos establecer una relación directa entre el número de operativos y el número de decomisos; sin embargo, asumimos que las diferencias en valores en cuanto a número de especies e individuos, entre 2005 y 2009 están probablemente relacionadas con el número de municipios, donde se realizaron los operativos de vigilancia y de control por año, ya que cuando se hace este análisis, observamos que para el 2009 se llevaron a cabo operativos en 13 municipios, mientras que para el 2005, solamente se registran para cuatro (Figura 1 ).

Decomisos por municipio. En términos de la cobertura de los decomisos para el departamento, se identificó que este procedimiento fue realizado en 18 de los 30 municipios del ente territorial (60\%), siendo Quibdó, con 486 individuos

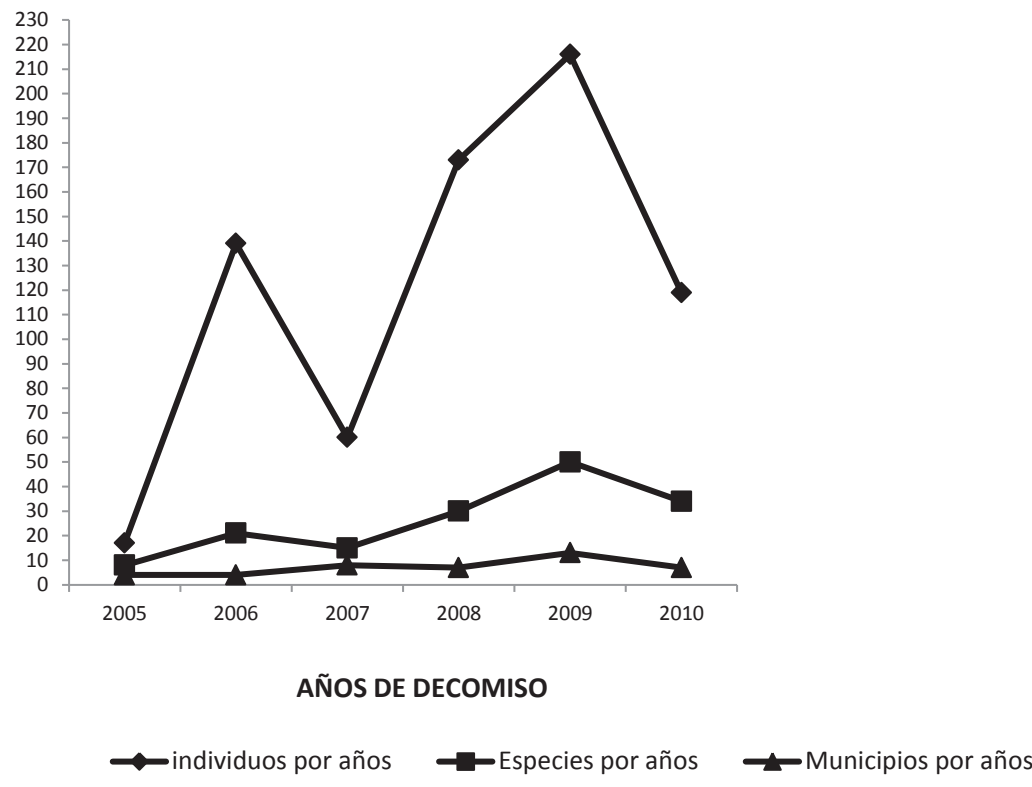

Fuente: Elaboración propia con datos de CODECHOCO, 2010.

Figura 1. Número de especies e individuos de fauna silvestre decomisados por año y número de municipios, donde se desarrollaron operativos por año. 
(67\%), el lugar donde se desarrolló la mayor cantidad de incautaciones de fauna silvestre. En consecuencia con lo anterior, también fue esta ciudad, la localidad donde se registró el mayor número de especies decomisadas (Figura 2).

La diferencia entre el número de individuos decomisados en el municipio de Quibdó y los demás municipios del departamento del Chocó es estadísticamente significativa $(\mathrm{F}=10.03$; $P=0.00 ; \alpha=0.05)$; esto, probablemente, se debe a que Quibdó es la ciudad capital del departamento y, por tanto, allí se encuentra situada la sede principal de CODECHOCO, como autoridad ambiental, que sumado a mayor presencia de las instituciones de apoyo (DAS, CTI, Policía Ambiental), permiten un mayor número de operativos para la vigilancia y el control del aprovechamiento de fauna silvestre en la región.

En cuanto al lugar de procedencia de los individuos decomisados por las autoridades ambientales en el departamento del Chocó, se identificó que el 75\% (543 individuos) eran provenientes de localidades del departamento y el 14\% (102 individuos) de otras ciudades de Colombia (Cali, Medellín y Pereira). Solamente se reporta un individuo proveniente de otro país (Panamá) y el 10,9\% restante son individuos cuya información del sitio de proveniencia no se encuentra registrada en las bases de datos de CODECHOCO.

A nivel del departamento, se encontró que los 543 individuos decomisados son procedentes de 24 de los 30 municipios del ente territorial. En este mismo sentido, el mayor reporte de procedencia por municipios se dio para Quibdó, con 301 individuos, que equivalen al 55\% del total registrado.

La relación entre el lugar de decomiso y los sitios de procedencia de los individuos, no evidencia la existencia de rutas regulares establecidas para el tráfico de fauna silvestre, desde el Chocó hacia otros lugares, pues la mayor cantidad de individuos se ha decomisado en el municipio de Quibdó y, según los datos de procedencia, han sido capturados en esta misma zona.

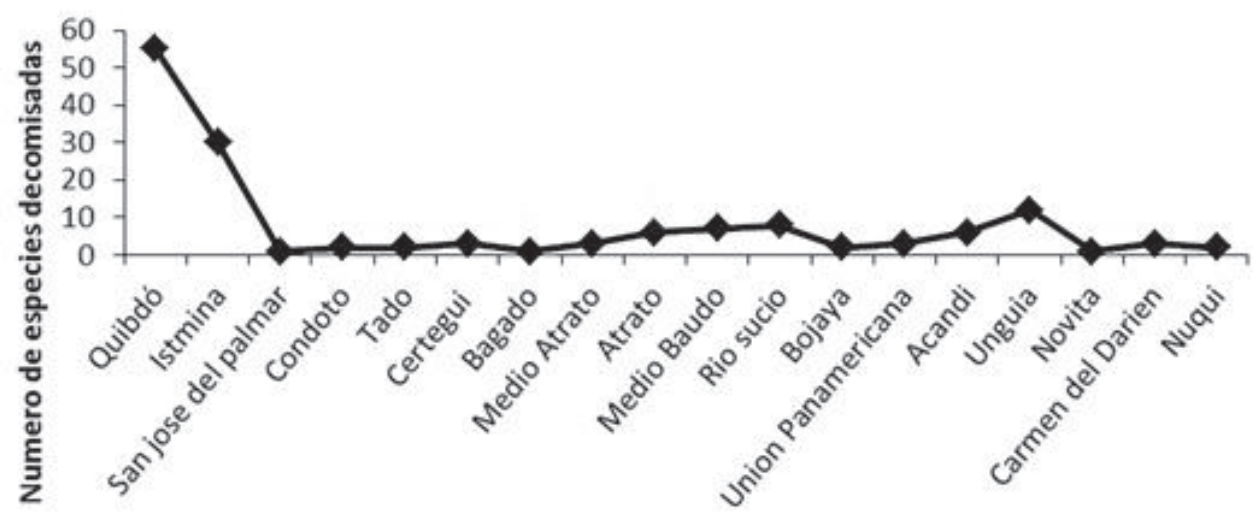

Municipio

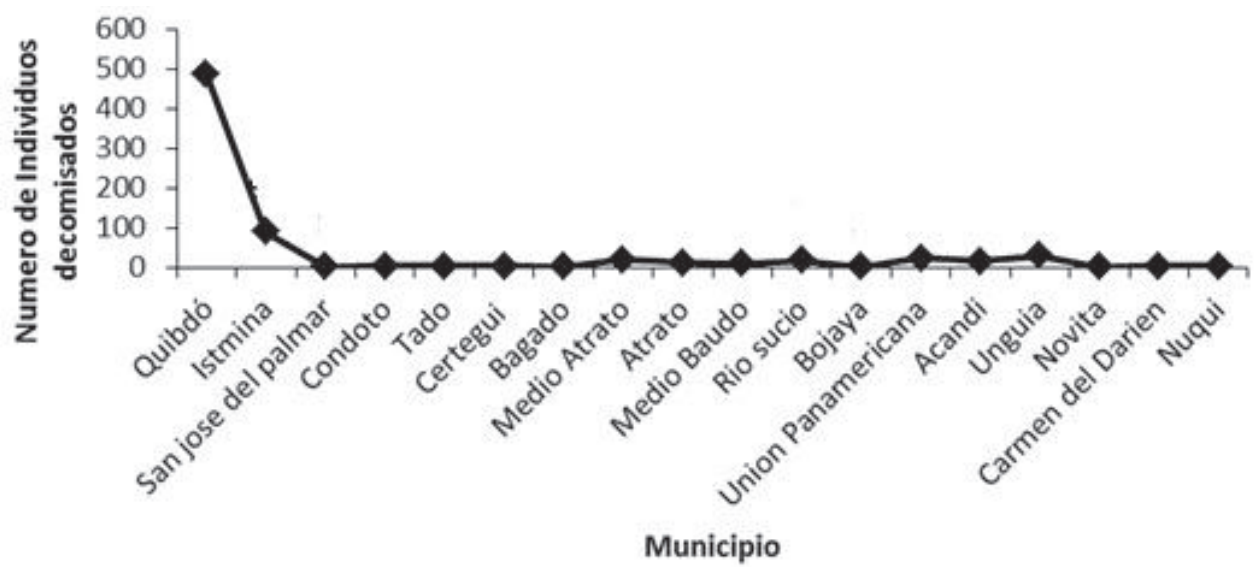

Fuente: Elaboración propia con datos suministrados por CODECHOCO, 2010.

Figura 2. Número de especies e individuos de fauna silvestre decomisados por municipio en el departamento del Chocó (2005 - 2010). 
La finalidad del porte de los ejemplares al momento del decomiso, no se registra en las estadísticas de las autoridades ambientales, por lo cual, en este estudio no se presenta un análisis al respecto.

Destino final de los individuos decomisados. Acorde con los parámetros establecidos en los protocolos para el manejo y la disposición de animales post-decomiso, expedidos por el Ministerio de Medio Ambiente Vivienda y Desarrollo Territorial, hoy Ministerio de Ambiente y Desarrollo Sostenible y la Resolución 2064 de 2010, el destino final de los individuos decomisados puede ser: liberación (dura y suave), reubicación, custodia y eutanasia.

La liberación dura, se refiere al destino final donde se regresa el individuo al medio natural de manera abrupta, sin permitirle ningún período de aclimatación; esto se realiza con individuos que estén física y comportalmente sanos. Se debe hacer siempre en la zona de distribución natural propia de la especie y, en lo posible, en la misma área geográfica. La liberación suave, se realiza con los animales que presentan algún cambio en su comportamiento silvestre, como por ejemplo, algún nivel de amansamiento. Estos animales requieren de algún tiempo de adaptación y de aclimatación, para poder ser regresados a su ambiente natural.

La reubicación, se produce cuando el individuo no se puede liberar y el Centro de Atención y Valoración (CAV), que mantiene al animal, no presenta las condiciones óptimas y necesarias para su tenencia. Generalmente, los individuos son reubicados en unidades con capacidades técnicas y de manejo adecuados, para la permanencia de ejemplares de fauna silvestre (zoocriaderos). La custodia se da cuando se le permite al infractor la tenencia temporal del animal, debido al estado de amansamiento del mismo. Finalmente, la eutanasia es una alternativa que se practica a los animales que no tienen ninguna posibilidad de sobrevivir o que física o biológicamente sean una amenaza para el ecosistema o las personas. Se debe realizar bajo protocolos, que garanticen la mínima probabilidad de dolor o de sufrimiento para el individuo sacrificado.
Para el departamento del Chocó, se encontró que posterior al decomiso de fauna silvestre, el principal destino final fue la liberación (dura), pues el $58 \%$ de los animales fueron regresados al ambiente natural, el 30,1\% fueron entregados en custodia a cazadores de la zona, el $9,7 \%$ se reubicaron en zoocriaderos del departamento y el 2,2\% fueron sacrificados (Tabla 3). Estos resultados difieren con los obtenidos por Cruz-Antía et al. (2010), en investigaciones en el departamento de Vichada (Puerto Carreño), donde la mayor parte de los individuos decomisados fueron dejados al tenedor en custodia, dado a que en inmediaciones de esta localidad no se cuenta con lugares apropiados para el mantenimiento de los mismos.

En cuanto a la dinámica del destino final post-decomiso por año, se identificó que las liberaciones tuvieron mayor importancia a partir de 2007, lo cual, probablemente, se debe a los problemas de orden público que se presentaban con mayor frecuencia en años anteriores. Dichos problemas limitaban los operativos de liberación en la selva y se optaba por la entrega en custodia a tenedores de fauna silvestre, que, por lo general, son los mismos cazadores de las comunidades. Otro hecho importante a destacar es el posible factor de cambio en la definición del destino final de los individuos decomisados, que generó, en el 2007, la implementación de los Centros de Atención y Valoración de Fauna Silvestre (CAV), que pese a su poca eficiencia en el departamento, han sido usados para el mantenimiento de algunos ejemplares posterior al decomiso, razón por la cual, se observa un aumento en el porcentaje de individuos reubicados para el 2008 y 2009 y el decrecimiento en esta materia para 2010; probablemente, se debe a que para esta fecha ya se había llegado a la totalidad de individuos que estos soportaban.

Estado de conservación de las especies decomisadas. El 80,6\% de las especies decomisadas en el departamento del Chocó, durante los seis años de evaluación, están ubicadas en alguna de las categorías de amenaza establecidas por la Unión Internacional para la Conservación de la Naturaleza (UICN), solamente un 19,4\% (equivalentes a 14 especies), no se reporta en dichos registros (Tabla 2).

Tabla 3. Porcentaje de individuos de fauna silvestre por destino final post-decomiso en el departamento del Chocó, para cada año de evaluación (2005-2010).

\begin{tabular}{|l|c|c|c|c|c|c|c|}
\hline Año/Destino & $\mathbf{2 0 0 5}$ & $\mathbf{2 0 0 6}$ & $\mathbf{2 0 0 7}$ & $\mathbf{2 0 0 8}$ & $\mathbf{2 0 0 9}$ & $\mathbf{2 0 1 0}$ & Total años de evaluación \\
\hline Liberado (\%) & 5,9 & 14,4 & 73,4 & 82,1 & 57,9 & 73,9 & 58,0 \\
\hline Reubicado (\%) & 0,0 & 0,0 & 0,0 & 2,3 & 30,5 & 0 & 9,7 \\
\hline Custodia (\%) & 94,1 & 83,5 & 23,3 & 13,9 & 8,8 & 24,4 & 30,1 \\
\hline Sacrificado (\%) & 0 & 2,2 & 3,3 & 1,7 & 2,8 & 1,7 & 2,2 \\
\hline TOTAL & 100 & 100 & 100 & 100 & 100 & 100 & 100 \\
\hline
\end{tabular}

Fuente: Elaboración propia con datos de CODECHOCO, 2010. 
En términos de las categorías de amenaza, se identificó que el $75,8 \%$ de las especies decomisadas se ubican en preocupación menor; el 8,6\%, en vulnerable; el 8,6\%, en peligro; el $6,8 \%$, casi amenazado (Tabla 4).

Agradecimientos: Se agradece especialmente a la Corporación Autónoma Regional para el Desarrollo Sostenible del Chocó (CODECHOCO) y al Instituto Colombiano de Desarrollo Rural (INCODER), por el suministro de información que fue fundamental para la realización de este estudio. A los miembros del Grupo de Investigación en Recursos Naturales para el Desarrollo Territorial de la Universidad Tecnológica del Chocó, por su gran apoyo en el desarrollo de la investigación. Conflictos de intereses: El manuscrito fue preparado y revisado con la participación de todos los autores, quienes declaramos que no existe ningún conflicto de intereses que ponga en riesgo la validez de los resultados presentados. Financiación: Este estudio fue financiado por la Universidad Tecnológica del Chocó "Diego Luís Córdoba”, a través de su Vicerrectoría de Investigación.

\section{BIBLIOGRAFÍA}

1. BAPTISTE, L.G.; POLANCO, R.; HERNÁNDEZ, S.; QUICENO, M.P. 2002. Fauna silvestre de Colombia: Historia económica y social de un proceso de marginalización. En: Ulloa, A. (ed.). Rostros culturales de la fauna. Las relaciones entre los humanos y los animales en el contexto colombiano. Bogotá: Inst. Col. Antropol. Historia. p.295-340.

Tabla 4. Porcentaje de especies de fauna silvestre decomisada en el departamento del Chocó, por categoría de amenaza, según la clasificación de la UICN.

\begin{tabular}{|c|c|c|c|c|c|c|c|}
\hline \multirow{2}{*}{$\begin{array}{c}\text { Catego- } \\
\text { rías }\end{array}$} & \multirow{2}{*}{ Descripción } & \multicolumn{6}{|c|}{ Especies por grupos taxonómicos de vertebrados } \\
\hline & & Peces & Reptiles & Aves & $\begin{array}{l}\text { Mamífe- } \\
\text { ros }\end{array}$ & Total & $\%$ \\
\hline $\begin{array}{l}\text { Preocu- } \\
\text { pación } \\
\text { menor } \\
\text { (LC) }\end{array}$ & $\begin{array}{l}\text { Un taxón está en la categoría de preo- } \\
\text { cupación menor cuando habiendo sido } \\
\text { evaluado, no cumple ninguno de los cri- } \\
\text { terios que definen las categorías estable- } \\
\text { cidas. }\end{array}$ & 0 & 1 & 25 & 18 & 44 & 76 \\
\hline $\begin{array}{c}\text { Casi ame- } \\
\text { nazado } \\
(\mathrm{NT})\end{array}$ & $\begin{array}{l}\text { Un taxón está en la categoría de casi } \\
\text { amenazado, cuando ha sido evaluado } \\
\text { según los criterios y no satisface, ac- } \\
\text { tualmente, los criterios para en peligro } \\
\text { crítico, en peligro o vulnerable, pero } \\
\text { está cercano a satisfacer los criterios o, } \\
\text { posiblemente, los satisfaga en un futuro } \\
\text { cercano. }\end{array}$ & 1 & 0 & 1 & 2 & 4 & 7 \\
\hline $\begin{array}{l}\text { Vulnerable } \\
\text { (VU) }\end{array}$ & $\begin{array}{l}\text { Es aquella especie amenazada que no } \\
\text { se encuentra en peligro inminente de ex- } \\
\text { tinción en el futuro cercano, pero podría } \\
\text { llegar a estarlo, de continuar la reduc- } \\
\text { ción de sus poblaciones naturales y el } \\
\text { deterioro de su área de distribución. }\end{array}$ & 0 & 2 & 1 & 2 & 5 & 9 \\
\hline $\begin{array}{c}\text { En peligro } \\
\text { (EN) }\end{array}$ & $\begin{array}{l}\text { Es aquella especie amenazada sobre } \\
\text { la que se cierne una alta probabilidad } \\
\text { de extinción en el estado silvestre en el } \\
\text { futuro cercano, en virtud de que existe } \\
\text { una tendencia a la reducción de sus po- } \\
\text { blaciones naturales y un deterioro de su } \\
\text { área de distribución. }\end{array}$ & 0 & 1 & 2 & 2 & 5 & 9 \\
\hline Total & & 1 & 4 & 29 & 24 & 58 & 100 \\
\hline
\end{tabular}


2. BENNETT, E.; ROBINSON, J. 2000. Hunting for sustainability in tropical forests. Columbia, New York: University Press. p.499-519.

3. CASTAÑO-MORA, O.V. 2002. Libro rojo de reptiles de Colombia. Serie Libros Rojos de Especies Amenazadas de Colombia. Instituto de Ciencias NaturalesUniversidad Nacional de Colombia, Ministerio del Medio Ambiente, Conservación Internacional-Colombia. Bogotá, Colombia. p.92-94.

4. CONTRALORÍA GENERAL DE LA REPÚBLICA. 2005. Comercio de bienes derivados de la vida silvestre. En: Mancera, N.J.; Reyes, O. (Eds.). Estado de los Recursos Naturales y del Ambiente 2004-2005 (Informe Anual al Congreso de la República de Colombia). Bogotá: Contraloría Delegada para el Medio Ambiente. p.111-213.

5. CRUZ, A.D.; GÓMEZ, J.R. 2010. Aproximación al uso y tráfico de fauna silvestre en Puerto Carreño, Vichada, Colombia. Revista P.U.J.B Ambiente y Desarrollo. p.10-11.

6. INCODER. 2010. Rendición de cuenta, informe de gestión Incoder 2010. Subgerencia de pesca y acuicultura. 107p

7. MAYR MALDONADO, J. 1999. Gestión ambiental para la fauna silvestre en Colombia. Rev. Acad. Colomb. Cienc. 23 (Supl.):712-715.

8. MINISTERIO DE AMBIENTE, VIVIENDA Y DESARROLLO TERRITORIAL. 2005. Resolución 0572 de 2005. Por el cual se modifica la Resolución No. 0584 del 26 de junio de 2002 y se adoptan otras determinaciones. 9p.

9. MINISTERIO DE AMBIENTE, VIVIENDA Y DESARROLLO TERRITORIAL. 2010. Resolución 2064 de 2010, por la cual se reglamentan las medidas posteriores a la aprehensión preventiva, restitución o decomiso de especímenes de especies silvestres de Fauna y Flora Terrestre y Acuática y se dictan otras disposiciones. Colombia. 86p.

10. MOJICA, J.I.; CASTELLANOS, C.; USMA, J.S.; ÁLVAREZ, R. 2002. Libro rojo de peces dulceacuícolas de
Colombia. Serie Libros Rojos de Especies Amenazadas de Colombia. Instituto de Ciencias Naturales - Universidad Nacional de Colombia y Ministerio del Medio Ambiente. Bogotá, Colombia. 285p.

11. RANGEL, J.O. 2004. Colombia diversidad biótica EL Chocó Biogeográfico. Costa pacífica IV Universidad Nacional de Colombia. Ed. Guadalupe. Bogotá. 1024p.

12. REDFORD, K.H. 1992. The empty forest. Biosci. 42(6):412-422.

13. REPÚBLICA DE COLOMBIA. 1993. Ley 99 de 1993 , mediante la cual se crea el Ministerio del Medio Ambiente, se reordena el Sector Público encargado de la gestión y conservación del medio ambiente y los recursos naturales renovables, se organiza el Sistema Nacional Ambiental, SINA, y se dictan otras disposiciones. Colombia. 59p.

14. REPÚBLICA DE COLOMBIA. 2009. Ley 1333 de 2009, mediante la cual se establece el procedimiento sancionatorio ambiental y se dictan otras disposiciones. Colombia. 19p.

15. ROBINSON, J.; REDFORD, K. 1991. Neotropical wildlife use and conservation. Chicago: University of Chicago Press. p.415-429.

16. RODRÍGUEZ MAHECHA, J.V.; ALBERICO, M.; TRUJILLO, F.; JORGENSON, J. 2006. Libro Rojo de los mamíferos de Colombia. Libros rojos de las especies amenazadas de Colombia. Conservación Internacional-Colombia, Ministerio del Medio Ambiente, Vivienda y Desarrollo Territorial. Bogotá, Colombia. 433p.

17. THORBJARNARSON, J.B.; LAGUEUX, C.; BOLZA, D.; KLEMENS, M.; MEYLAN, A. 2000. Human use of turtle: a worldwide perspective. In: Klemens, M.W. (ed.). Turtle conservation. Smithsonian Institution Press. Washington and London. p.33-84.

Recibido: Abril 27 de 2012

Aceptado: Noviembre. 8 de 2012 